\title{
Partizipatives Sozialmanagement
}

\author{
Martin Stummbaum und Margit Stein
}

\begin{abstract}
Rund drei Millionen Menschen in Deutschland engagieren sich in Selbsthilfegruppen. Am Beispiel der pflegebezogenen Betroffenenselbsthilfe kann gezeigt werden, wie Wohlfahrtsverbände und soziale Leistungserbringer mit solchem Engagement kooperieren können.
\end{abstract}

Selbsthilfegruppierungen schicken sich an, Wohlfahrtsverbänden und anderen Organisationen zunehmend den Rang als intermediäre Instanzen abzulaufen (vgl. Brömme und Strasser 2000). Mit dem Pflegeversicherungs-Weiterentwicklungsgesetz (§ 45d SGB XI - vgl. Kasten) erhält dieser Trend eine weitere gesetzliche Grundlage. Gleichzeitig eröffnet diese Gesetzesnovelle innovative Perspektiven, intermediäre Potenziale aus einem sektorenübergreifenden Zusammenwirken von (pflegebezogenem)

Selbsthilfeengagement und professionellen Leistungsangeboten zu generieren. Intermediäre Potenziale (wohlfahrtsverbandlicher) Organisationen können in solchen Arrangements eine Revitalisierung erfahren.

Im Jahre 2004 engagierten sich in Deutschland circa drei Millionen Bürgerinnen und Bürger in rund 100.000 Selbsthilfegruppen (Robert Koch Institut 2004). Diese Erfolgsstatistik basiert überwiegend auf der gesundheitsbezogenen Selbsthilfe. Der Anteil gesundheitsbezogener Selbsthilfegruppierungen in der deutschlandweiten Selbsthilfelandschaft wird auf 70 Prozent geschätzt (vgl. Robert Koch Institut 2004).

Im Vergleich zur Gesundheitsselbsthilfe, die vielfach als »vierte Säule« des deutschen Gesundheitswesens tituliert wird (vgl. Keidel 2008), konnte sich eine dezidierte Pflegeselbsthilfe (noch) nicht entsprechend konturieren und etablieren. Dennoch ist die Herausforderung und Problematik von Pflege in der deutschen Selbsthilfegruppenlandschaft nicht als eine randständige Thematik zu betrachten. Die Herausforderungen und Problematiken von Pflege stellen eine oftmals hintergründige Querschnittsthematik dar, welche vor allem in gesundheitsbezogenen Selbsthilfegruppen wie beispielsweise von Alzheimer Erkrankten, Eltern mehrfach behinderter Kinder oder Patientinnen und Patienten nach dem Schlaganfall als ein Bewältigungsaspekt von Krankheits- oder Unfallfolgen (mit-) behandelt wird.

Das Statistische Bundesamt weist in der Pflegestatistik 2005 rund 2,6 Prozent der Bevölkerung als pflegebedürftig aus. Neben diesen im Sinne des Pflegeversicherungsgesetzes insgesamt 2,13 Millionen pflegebedürftigen Menschen existieren weitere statistisch nicht erfasste Pflegebedürftige, deren Pflegebedarf nach dem Pflegever-

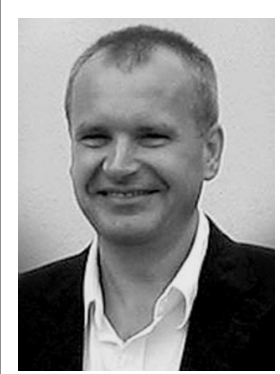

Martin Stummbaum ist wissenschaftlicher Mitarbeiter und Doktorand am Lehrstuhl für Sozialpädagogik und Gesundheitspädagogik der Katholischen Universität Eichstätt-Ingolstadt. Seine inhaltlichen Arbeitsschwerpunkte liegen u. a. im Bereich des Sozial- und Gesundheitsmanagements sowie der Selbsthilfe (-unterstützung). E-Mail martin.stummbaum@ku-eichstaett.de

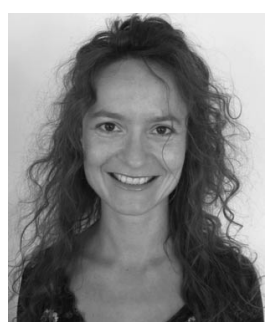

Prof. Dr. phil. habil. Margit Stein ist Hochschullehrerin für Klinische Sozialarbeit und Rehabilitation im Studiengang Gesundheitswesen und Sozialwesen an der Fachhochschule Nordhausen und Privatdozentin am Lehrstuhl für Sozialpädagogik und Gesundheitspädagogik der Katholischen Universität Eichstätt-Ingolstadt. Ihre inhaltlichen Arbeitsschwerpunkte liegen u. a. in der Werte- und Selbsthilfeforschung. E-Mail stein@fh-nordhausen.de

sicherungsgesetz nicht anerkannt wurde oder die ihren Pflegebedarf nicht pflegegesetzlich geltend gemacht haben (Pfaff 2008).

Über die grundsätzliche Bereitschaft der beiden Bevölkerungsgruppen der Pflegebedürftigen und deren Angehörigen, sich in Selbsthilfegruppierungen zu engagieren, können aufgrund der uneinheitlichen Ergebnislage vorhandener Erhebungen nur Spekulationen angestellt werden (vgl. Robert Koch Institut 2004).

Unter Zugrundelegung des vom Robert-Koch-Institut im Jahre 2003 fernmündlich durchgeführten Gesundheitssurveys, nach dem neun Prozent der Befragten im Alter von 18 bis 79 Jahren aus (in-) direkter Betroffenheit an einer Selbsthilfegruppe teilgenommen haben oder noch teilnehmen (Robert Koch Institut 2004), beläuft sich das Engagementpotenzial von pflegebezogenen Selbsthilfegruppen (für Pflegebedürftige und deren Angehörigen) auf circa 400.000 Personen.

Die Autoren dieses Beitrags (Stummbaum und Stein 2008) legten für eine differenziertere Erhebung von individuellem und kollektivem Selbsthilfeengagement eine 
theoretische Folie zugrunde, die sich aus einer Verschränkung von Sinn- und Werteprozessen entfaltet. Die theoretische Kontextuierung dieses Erhebungsansatzes basiert auf den Arbeiten von Aron Antonovsky (1997) zur Salutogenese und von Shalom Schwartz (1992) zur Wertorientierung. Diese aus Sinn- und Werteprozessen gespannte Theoriefolie bildet einen zusammenhängenden Hintergrund $\mathrm{ab}$, auf dem $\mathrm{u}$. a. individuelle Potenziale und Arrangementformen kollektiven Betroffenenselbsthilfeengagements dargestellt werden können. Erste empirische Erhebungen zur gesundheitsbezogenen Betroffenenselbsthilfe sind bereits in Arbeit (Stummbaum/Stein 2008). Zur pflegebezogenen Betroffenenselbsthilfe ist eine diesbezügliche Erhebung im Rahmen eines Modellvorha-

\section{Förderung ehrenamtlicher Strukturen sowie der Selbsthilfe}

(1) In entsprechender Anwendung des § 45c können die dort vorgesehenen Mittel des Ausgleichsfonds, die dem Spitzenverband Bund der Pflegekassen zur Förderung der Weiterentwicklung der Versorgungsstrukturen und Versorgungskonzepte insbesondere für demenziell Erkrankte zur Verfügung stehen, auch verwendet werden zur Förderung und zum Auf- und Ausbau

1.

von Gruppen ehrenamtlich tätiger sowie sonstiger zum bürgerschaftlichen Engagement bereiter Personen, die sich die Unterstützung, allgemeine Betreuung und Entlastung von Pflegebedürftigen, von Personen mit erheblichem allgemeinem Betreuungsbedarf sowie deren Angehörigen zum Ziel gesetzt haben, und

2. von Selbsthilfegruppen, -organisationen und -kontaktstellen, die sich die Unterstützung von Pflegebedürftigen, von Personen mit erheblichem allgemeinem Betreuungsbedarf sowie deren Angehörigen zum Ziel gesetzt haben.

(2) Selbsthilfegruppen im Sinne von Absatz 1 sind freiwillige, neutrale, unabhängige und nicht gewinnorientierte Zusammenschlüsse von Personen, die entweder auf Grund eigener Betroffenheit oder als Angehörige das Ziel verfolgen, durch persönliche, wechselseitige Unterstützung, auch unter Zuhilfenahme von Angeboten ehrenamtlicher und sonstiger zum bürgerschaftlichen Engagement bereiter Personen, die Lebenssituation von Pflegebedürftigen, von Personen mit erheblichem allgemeinem Betreuungsbedarf sowie deren Angehörigen zu verbessern. Selbsthilfeorganisationen im Sinne von Absatz 1 sind die Zusammenschlüsse von Selbsthilfegruppen nach Satz 1 in Verbänden. Selbsthilfekontaktstellen im Sinne von Absatz 1 sind örtlich oder regional arbeitende professionelle Beratungseinrichtungen mit hauptamtlichem Personal, die das Ziel verfolgen, die Lebenssituation von Pflegebedürftigen, von Personen mit erheblichem allgemeinem Betreuungsbedarf sowie deren Angehörigen zu verbessern.

(3) § 45c Abs. 6 Satz 4 gilt entsprechend.

§ 45d der Sozialen Pflegeversicherung (SGB XI) bens der Fachhochschule Nordhausen im Innovationsbereich der niederschwelligen unabhängigen Pflegeberatung anvisiert (Hey/Naumann/Stein 2009).

Traditionell lässt sich hinsichtlich der Arrangementformen zwischen Selbsthilfegruppen, Selbsthilfeorganisationen und Selbsthilfeinitiativen differenzieren (vgl. Borgetto 2004 , S. 81 ff.). Je nach Arrangementform ergeben sich unterschiedliche Bedingungs- und Perspektivenkontexte. Stummbaum (2008) clustert das intermediäre und marktwirtschaftliche Perspektivenspektrum einer Bezugnahme von (wohlfahrtsverbandlichen) Organisationen auf Selbsthilfegruppen in vier Aufgabenbereiche des strategischen Sozialmanagements.

\section{Kooperationsformen}

Im Kontext pflegebezogener Betroffenenselbsthilfe offeriert insbesondere eine Bezugnahme auf Selbsthilfeinitiativen vielversprechende Perspektiven. Selbsthilfeinitiativen als eine im Vergleich zu Selbsthilfegruppen formalisiertere Form von Selbsthilfeengagement können ein Arrangement bilden, das u. a. den Koproduktionsprozess von Pflegeleistungen befördert, pflegende Angehörige entlastet und die (Kunden-) Souveränität ihrer Mitglieder stärkt. Pflegebezogene Selbsthilfeinitiativen können analog des erfolgreichen und etablierten Modells der Eltern-Kind-Selbsthilfeinitiativen - einen zentralen Beitrag zur Umsetzung der mit dem Pflegeversicherungs-Weiterentwicklungsgesetz verfolgten Intention einer an den Bedürfnissen und Wünschen der Pflegebedürftigen und deren Angehörigen ausgerichteten Pflege leisten.

Exemplarisch seien an dieser Stelle drei mögliche Modelle einer pflegespezifischen Adaption von Eltern-Kind-Selbsthilfeinitiativen aufgeführt:

- Adaptionsmodell A: Selbsthilfeinitiativen zur selbstorganisierten Leistungserbringung, beispielsweise Pflegebedürftigen-Angehörigen-Selbsthilfeinitiative zur ambulanten Pflege und Betreuung mit oder ohne Hinzuziehung von angestellten oder beauftragten Arbeitskräften.

- Adaptionsmodell B: Selbsthilfeinitiativen zum selbstorganisierten Leistungseinkauf, beispielsweise Selbsthilfeinitiative »Eichstätter Senior/innen mit Pflegebedarf für einen günstigeren und bedarfsgerechten Einkauf von »Essen auf Rädern«.

- Adaptionsmodell C: Selbsthilfeinitiativen für selbstorganisierte Trägerschaften, beispielsweise Selbsthilfeinitiative »Nordhausener Seniorenwohngemeinschaft«.

Die genannten Modellbeispiele pflegebezogener Selbsthilfeinitiativen können in unterschiedlichen Bezugskontexten zu (wohlfahrtsverbandlichen) Organisationen der Pflege und Sozialen Arbeit stehen. Gemeinhin befinden sich Selbsthilfegruppierungen und (wohlfahrtsverbandliche) Organisationen in ihren Leistungsangeboten in keinen immediaten oder integrativen Bezügen. Vor dem Hintergrund gerechter Teilnahmechancen an (pflegebezogenen) Selbsthilfegruppen (vgl. Borgetto/Kolba 2008) erscheinen solche unmittelbaren und integrativen Leistungssettings dringend angeraten (vgl. Stummbaum 2009). 
Auch darf in diesem Zusammenhang nicht vergessen werden, dass Selbsthilfegruppenengagement nicht nur mit positiven, sondern auch mit negativen Folgen verbunden sein kann. Vogel (1990) erfasste in einer qualitativen Studie die negativen Folgen von Selbsthilfegruppenengagement und kam zu dem Ergebnis, dass ein Scheitern von Betroffenenselbsthilfeengagement mit erheblichen negativen Nachwirkungen verbunden sein kann. Nachdem sich in den letzten Jahren auch bei Leiterinnen und Leitern von Selbsthilfegruppen negative Nebenwirkungen, die sich unter dem Syndrom »Burnout aufgrund von Selbsthilfegruppenengagement « subsumieren lassen, gehäuft haben (vgl. Internet http://www.agus-selbsthilfe.de und Schulte 2005), erscheint bei der Konturierung und Etablierung einer pflegebezogenen Betroffenenselbsthilfe das Verlassen tradierter Wege angezeigt (vgl. Kreling 2008, Stummbaum/Birgmeier 2009 und Stummbaum 2009).

Richtungsweisende Hinweise für das Beschreiten innovativer Wege können beispielsweise aus dem Modellprojekt des Selbsthilfezentrums München und dem Krankenhaus Neuperlach zur professionellen Begleitung einer Selbsthilfegruppe für Schmerzpatienteninnen und Schmerpatienten (Hammerl \& Hermes 2002) gewonnen werden. Im Rahmen dieses Modellprojekts wurden interessierte Patientinnen und Patienten auf dem Weg zu einer funktionierenden Selbsthilfegruppe von professionellen Fachkräften begleitet und unterstützt (vgl. auch Reichwald 1999).

\section{Resümee}

Mit dem Pflegeversicherungs-Weiterentwicklungsgesetz sind Grundlagen für das Beschreiten innovativer Wege zu immediaten und integrativen Leistungssettings geschaffen worden. Vonseiten (wohlfahrtsverbandlicher) Organisationen können solche Wege beispielsweise mittels partizipativer Sozialmanagementansätze geebnet werden.

Partizipatives Sozialmanagement fördert immediate und integrative Leistungssettings, indem Betroffenenselbsthilfe an Planungs-, Entscheidungs- und Leistungsprozessen (wohlfahrtsverbandlicher) Organisationen der Pflege und Sozialen Arbeit beteiligt und aktiv eingebunden werden. Partizipatives Sozialmanagement überschreitet Organisationsgrenzen und Ansätze bruchstückhafter und additiver Leistungssettings. Partizipatives Sozialmanagement bereitet nicht für, sondern mit Betroffenenselbsthilfegruppierungen immediate und integrative Leistungssettings, in denen die Ressourcen von Fremd- und Selbsthilfe bewältigungsoptimale Synergien entwickeln können.

Partizipatives Management revitalisiert in diesem skizzierten Zusammenwirken (wohlfahrtsverbandlicher) Organisationsleistung mit Betroffenenselbsthilfeengagement intermediäre Potenziale.

\section{Literatur}

Antonovsky, A. (1997): Salutogenese - Zur Entmystifizierung der Gesundheit. Tübingen.

Borgetto, B. \& Kolba, N. (2008): Wie anfällig ist die gemeinschaftliche Selbsthilfe für die Reproduktion und Produktion sozialer und gesundheitlicher Ungleichheit? In: Bauer, U. \& Büscher, A. (Hg.): Soziale Ungleichheit und Pflege. Beiträge sozialwissenschaftlich orientierter Pflegeforschung, Gesundheit und Gesellschaft. Wiesbaden.

Borgetto, B. (2004): Selbsthilfe und Gesundheit - Analysen, Forschungsergebnisse und Perspektiven in der Schweiz und in Deutschland. Bern.

Brömme, N. \& Strasser, H. (2000): Exklusive Solidarität. Die ungleichen Folgen des Strukturwandels von Engagement und Partizipation, Tagungsbeitragsdokumentation: 30. Kongress der Deutschen Gesellschaft für Soziologie. Köln.

Hammerl, V. \& Hermes, K. (2002): Kooperation Krankenhaus und Selbsthilfe. Evaluation eines Modellprojekts. (Hg.): Selbsthilfezentrum München und Krankenhaus Neuperlach. München.

Hey, G. \& Naumann, S. \& Stein, M. (2009): Modellkonzeption zur Niederschwelligen unabhängigen Pflegeberatung. Nordhausen.

Keidel, T. (2008): Selbsthilfe - von der Protestbewegung bis zur vierten Säule im Gesundheitswesen. In: Die Demokratische Schule. DDS 2008-12. München.

Kreling, E. (2008): Qualität in der Selbsthilfe. Wie sich das Angebot von Supervision auf die Qualität der Gruppenarbeit auswirkt. In: Deutsche Arbeitsgemeinschaft Selbsthilfegruppen e. V.: Selbsthilfegruppenjahrbuch 2008. Gießen.

Pfaff, H. (2008): Pflegebedürftige heute und in Zukunft. In: Statistisches Bundesamt Deutschland (Hg.): STATmagagzin 11/2008. Wiesbaden.

Reichwald, U. (1999): Selbsthilfe und Psychologie. Ein Erfahrungsbericht aus der Arbeit mit Selbsthilfegruppen chronischer Schmerzpatienten. In: Deutsche Arbeitsgemeinschaft Selbsthilfegruppen e. V. (Hg.): Selbsthilfegruppenjahrbuch 1999. Gießen.

Robert Koch Institut (Hg.) (2004). Selbsthilfe im Gesundheitsbereich. Gesundheitsberichterstattung des Bundes (Heft 23). Berlin.

Schulte, H. (2005): Wer immer nur gibt, muss auch auftanken. Erfahrungen aus Seminaren der Frauenselbsthilfe nach Krebs. In: Deutsche Arbeitsgemeinschaft Selbsthilfegruppen e. V.: Selbsthilfegruppenjahrbuch 2005. Gießen.

Schwartz, S. H. (1994): Are there universal aspects in the structure and contents of human values? Journal of Social Issues. 50. 19-45.

Stummbaum, M. (2009): Soziale Arbeit im Spannungsfeld zwischen Marktökonomie und gruppenbezogener Betroffenenselbsthilfe. Eichstätt (im Erscheinen).

Stummbaum, M. (2008): Selbsthilfegruppen als strategischer Partner. In: SOZIALwirtschaft - Zeitschrift für Sozialmanagement 6/2008. S. 27-29.

Stummbaum, M. \& Stein, M. (2008): Selbsthilfeengagement konstituierende Sinn- und Werteprozesse. Eichstätt (unveröffentlichte Konzeption zur Erhebung »Sinn- und Werteprozesse in Selbsthilfegruppen«).

Vogel, R. (1990): Gesprächs-Selbsthilfegruppen. Interviews mit Aussteigern und Dabeigebliebenen. Berlin.

Internet http://www.agnus-

selbsthilfe.de/index.php?id=17 (2008) Zum Rücktritt der Leiterin der Selbsthilfegruppen »Angehörige um Suizid « (aufgerufen am 18.11.2008). 